\title{
Der psychotechnologische Komplex - Die Automatisierung mentaler Prozesse als demokratietheoretisches Problem
}

\author{
Bernd Bösel (iD
}

Angenommen: 29. Juli 2021 / Online publiziert: 25. August 2021

(C) Der/die Autor(en) 2021

Zusammenfassung Die Digitalisierung umfasst neben vielen anderen Aspekten auch die Automatisierung psychischer Prozesse. Damit wird allerdings ein wesentlicher Aspekt für ein demokratisches und progressives Politikverständnis unterlaufen. Der Artikel setzt sich mit der Verflechtung von Psyche, Technik und Politik zunächst anhand einer Diskussion des französischen Philosophen Bernard Stiegler (1952-2020) auseinander, der in seinen späteren Arbeiten Medien als Psychotechnologien zu konzipieren begann und dafür plädierte, die seit Foucault in vielen Disziplinen häufig gebrauchte Kategorie der Biomacht um diejenige der „Psychomacht“ zu ergänzen. Der in dieser Diskussion ebenfalls aufblitzende Begriff „Psychopolitik“ wird anschließend mit zwei deutschsprachigen Theoretiker*innen, Alexandra Rau und Byung-Chul Han, konkretisiert. Auf dieser begrifflichen wie zeitdiagnostischen Grundlage wird in der zweiten Hälfte des Artikels das ,psychotechnologische Arsenal" der Gegenwart umrissen: Gemeint sind damit jene digitaltechnologischen Innovationen, die auf die Beeinflussung psychischer Prozesse von Nutzer*innen abzielen, und zu denen man u.a. persuasive technologies, Micro-Targeting, Apps für Wellness und psychische Gesundheit sowie affective computing zählen kann. All diese Entwicklungen erzwingen zum Schluss eine Auseinandersetzung mit der vielfach beobachteten Rückkehr des Behaviorismus unter datenwissenschaftlichem Vorzeichen sowie mit der Frage, wie die Fähigkeit zu politischem Handeln innerhalb dieser Konstellation noch bewahrt werden kann, bevor der im Entstehen begriffene ,psychotechnologische Paternalismus“ die psychische Kapazitäten zur freien Deliberation und vor allem auch zur politischen Imagination völlig außer Gefecht setzt.

Schlüsselwörter Psychomacht · Psychopolitik · Behaviorismus · Paternalismus · Imagination

Bernd Bösel ( $\triangle)$

Institut für Künste und Medien, Universität Potsdam, Potsdam, Deutschland

E-Mail: bernd.boesel@uni-potsdam.de 


\title{
The psychotechnological complex-the automatization of mental processes as a problem for theories of democracy
}

\begin{abstract}
In addition to many other aspects, digitization also includes that of an automation of psychic processes. However, this undermines an essential aspect for a democratic and progressive understanding of politics. The article deals with the intertwining of psyche, technology, and politics first by means of a discussion of French philosopher Bernard Stiegler (1952-2020), who, in his later works, began to conceptualize media as psychotechnologies and argued for supplementing the category of biopower, so popularly used in many disciplines since Foucault, with that of "psychopower". The term "psychopolitics", which also emerges in this discussion, will then be given a more concrete meaning with the help of German theorists Alexandra Rau and Byung-Chul Han. On this conceptual as well as contemporary diagnostic basis, the second half of the article outlines the "psychotechnological arsenal" of the present: this refers to digital technological innovations that aim to influence the psychological processes of users, and to which one can count persuasive technologies, microtargeting, apps for wellness and mental health, and affective computing, among others. In conclusion, all these developments force us to deal with the often observed return of behaviorism under the sign of data science, as well as with the question of how political difference can still be preserved within this constellation, before the emerging "psychotechnological paternalism" completely disables the psychological capacities for free deliberation and, above all, for political imagination.
\end{abstract}

Keywords Psychopower · Psychopolitics · Behaviorism · Paternalism · Imagination

\section{Einleitung}

Die Frage, von welchen Bedingungen politisches Handeln abhängt, ist klarerweise eine für die politische Theorie zentrale. Dass diese Bedingungen wandelbar sind, erschwert ihre Beantwortung ungemein. Gerade in Zeiten, in denen wirtschaftliche, technologische und nun auch ökologische Disruptionen in immer höherer Taktung auftreten, ist eine Auseinandersetzung mit den Veränderungen dieser Bedingungen unausweichlich. „Menschen sind bedingte Wesen, weil ein jegliches, womit sie in Berührung kommen, sich unmittelbar in eine Bedingung ihrer Existenz verwandelt", schrieb Hannah Arendt (2001, S. 18) zu Beginn von Vita activa. Ausdrücklich hielt sie fest, dass die von Menschen selbstgeschaffenen Dinge ,die gleiche bedingende Kraft besitzen wie die bedingenden Dinge der Natur“ (ebd., S. 19). Arendt meint damit ausdrücklich auch technische Artefakte, deren Gebrauch im Extremfall zum Katalysator eines Epochenwandels wird, wie sie es für das Teleskop in Galileis Händen geltend macht (S. $329 \mathrm{ff}$.) Das politische Denken Arendts ist hier für die Medientheorie höchst anschlussfähig, geht diese doch ebenfalls davon aus, dass neue Medien in jeweils genau zu bestimmendem Ausmaß das psychosoziale und kognitive Gefüge rekonfigurieren, in dem sie jeweils in Erscheinung treten. 
Nun war zu Arendts Lebzeiten unmöglich absehbar, welche Ausmaße die Digitalisierung annehmen würde. Die Frage nach der Möglichkeit des politischen Handelns in der „digitalen Konstellation“ (Berg et al. 2020) muss, Arendts Vorgabe folgend, die Agentialität der digitalen Technologien in den Blick nehmen, die den Spielraum für die Politik und das Politische verschieben. Sie muss, anders gesagt, die „technologische Bedingung“ (Hörl 2011) mitreflektieren, insbesondere wenn diese Bedingung das verändert, was unter Diskurs, Öffentlichkeit und Freiheit verstanden wird. Bislang wurden diese Auswirkungen gerade von der Demokratietheorie noch nicht ausreichend berücksichtigt, auch wenn der Medienwandel durch die digitale Transformation zumindest so einen starken Umbruch bedeuten dürfte wie der einst durch die Erfindung des Buchdrucks eingeläutete, wie Borucki und Oswald (2020, S. 4) zurecht zu bedenken geben. Umso mehr gilt dies, wenn die Digitalisierung nicht nur die Art und Weise rekonfiguriert, in der bereits konstituierte politische Subjekte an öffentlichen Debatten teilnehmen, sondern darüber hinaus in die Konstitution politischer Subjekte eingreift - und das heißt auch, wenn nicht vor allem, in ihre psychischen Fähigkeiten, Neigungen und Dispositionen.

Von welchen psychischen Bedingungen hängt also politisches Handeln $a b$ ? Neben basalen Fähigkeiten wie diejenigen zur Wahrnehmung, Erinnerung, zum Sprechen und Denken spielen das Begehren und die Imagination eine eminent politische Rolle (Govrin 2020). Zudem muss so etwas wie Willensfreiheit gegeben sein oder zumindest unterstellt werden, denn sonst ließe sich nicht ernsthaft von politischer Willensbildung sprechen. Ob ein solcher Wille dann die konstitutiven Voraussetzungen findet, um sich auch ungehindert auszudrücken, steht auf einem anderen Blatt. Und selbstverständlich reicht es nach der Kritik am autonomen Subjektmodell der europäischen Neuzeit nicht mehr, einfach ein freies Subjekt mit authentischem, eigenem Willen zu postulieren. Anstatt nur von internen Bedingungen auszugehen, die einen freien Willen konstituieren, betont Beate Rössler (2017, S. 49) die Notwendigkeit auch externer Bedingungen, nämlich die soziale Umgebung, aus der viele Wünsche und Überzeugungen herrühren, die eine Person haben mag. Von dieser Anerkennung externer Bedingungen ist der Schritt zu der Auffassung nicht fern, dass das Subjekt und seine Vermögen immer schon von Sozialisierungs- und Erziehungsprozessen mitkonstituiert werden. Dass aber diese Subjektivierung, wie der gängige Terminus hierfür seit den Arbeiten Foucaults lautet, zugleich auch von Infrastrukturen und technischen Medien abhängt, eröffnet eine weitere unerlässliche Reflexionsebene.

Demokratische Gesellschaften tragen permanent das Risiko in sich, dass eine Mehrheit sich für die Einschränkung, Rücknahme oder Abschaffung der Demokratie entscheiden könnte. Der Selbsterhalt der Demokratien hängt mit davon ab, dass ihre Bürger*innen fähig und willens sind, ihren Wert hochzuhalten und die Demokratie erhaltende Entscheidungen zu treffen. Daher müssen Demokratien Interesse an der Formung von Subjekten haben, die willens und fähig sind, demokratiepolitisch zu agieren. Zugleich wird mit dem Willen zur politischen Konstitution der Subjekte deren Freiheit eingeschränkt. Mit dieser inneren Spannung müssen Demokratien umgehen können: Sie kommen ohne Subjektivierungsprogrammatiken nicht aus, die aber zugleich die Schwelle zur Indoktrination nicht überschreiten dürfen. Die Grenze zwischen Subjektivierung und Indoktrination ist allerdings schwer auszumachen. Hinzu kommt, dass auch in starken Demokratien gegenläufige Subjektivierungs- 
effekte zu finden sind, die antidemokratisch wirken. So mehren sich seit einigen Jahren Analysen, denen zufolge die technologische Bedingung zunehmend Einfluss auf die Formierung von Subjekten nimmt, und zwar durchaus zum Schaden demokratischer Prozesse (vgl. Oswald 2020, S. 332). Gerade die Schnelligkeit, mit der disruptive Entwicklungen über Gesellschaften hereinbrechen, schafft einen enormen Druck, allfällige demokratiegefährdende Effekte überhaupt erst einmal angemessen zu analysieren. Welchen Effekt haben also die digitalen Technologien auf die demokratische Subjektivierung? Wie verträgt sich insbesondere die Technisierung und Automatisierung psychischer Prozesse mit dem Ziel mindestens des Erhalts, nicht wenn sogar der Ausweitung der Demokratie?

Im Folgenden werden rezente Theorieangebote betrachtet, die der Verflechtung von Technik, Psyche und Politik besondere Aufmerksamkeit schenken und Begriffe schaffen, um jeweils entscheidende Aspekte im Hinblick auf demokratiepolitische Konsequenzen zu benennen. Zunächst werden einige Theoreme des französischen Philosophen Bernard Stiegler diskutiert, der in seinen späteren Arbeiten Medien als Psychotechnologien zu konzipieren begann und dafür plädierte, die seit Foucault in vielen Disziplinen häufig gebrauchte Kategorie der Biomacht um diejenige der Psychomacht zu ergänzen. Der hierbei ebenfalls aufblitzende Begriff Psychopolitik wird anschließend mit zwei deutschsprachigen Theoretiker*innen, Alexandra Rau und Byung-Chul Han, einer konkreteren Bedeutung zugeführt. Auf dieser begrifflichen wie zeitdiagnostischen Grundlage wird in der zweiten Hälfte des Artikels das psychotechnologische Arsenal der Gegenwart umrissen: Damit sind jene digitaltechnologischen Innovationen gemeint, die auf die Beeinflussung psychischer Prozesse von Nutzer*innen abzielen, und zu denen u. a. persuasive technologies, Micro-Targeting, Apps für Wellness und psychische Gesundheit sowie affective computing gezählt werden können. All diese Entwicklungen erzwingen zum Schluss eine Auseinandersetzung mit der vielfach beobachteten Rückkehr des Behaviorismus unter datenwissenschaftlichem Vorzeichen sowie mit der Frage, wie demokratisches Handeln innerhalb dieser Konstellation noch bewahrt bleiben kann, bevor der im Entstehen begriffene psychotechnologische Paternalismus die psychischen Kapazitäten zur freien Deliberation und vor allem auch zur politischen Imagination völlig außer Gefecht setzt.

\section{Generalisierte Proletarisierung und Psychomacht}

Für Bernard Stiegler waren die Entwicklungen, die mit der digitalen Grammatisierung menschlicher Existenz verbunden sind, weniger mit dem Versprechen der Demokratisierung verbunden als mit der Gefahr, dass sie zur Zerstörung der Psyche führen. ${ }^{1}$ So drastisch lautet tatsächlich seine in vielen Aussagen wiederholte Schlussfolgerung, und sie offenbart auch sogleich das erste Problem, das Stieglers Arbeiten jedem Ansinnen auf eine sachliche Rezeption stellen: seine oftmals superlativische

\footnotetext{
1 Im Sommer 2020 verstarb Bernard Stiegler gänzlich unerwartet. Sein ehemaliger Schüler Yuk Hui hat einen lesenswerten Nachruf geschrieben: https://networkcultures.org/geert/2020/08/09/yuk-hui-inmemory-of-bernard-stiegler/.
} 
und katastrophische Rhetorik. Eine wohlwollende Lektüre muss viele der detailblinden Zuspitzungen, denen man in seinen Texten begegnet, auf ein produktives Maß heruntermodulieren. Das zweite Problem ist die Terminologie: Oftmals lesen sich Stiegler-Passagen wie redundante Umarrangierungen der immer gleichen Neologismen, nach deren Definition man lange suchen muss, weil Stiegler seine vielen Bücher kaum systematisch durchgearbeitet hat. Einen konsistenten Begriffsgebrauch wird man ihm allerdings, sofern man seine Terminologie einmal verinnerlicht hat, nicht absprechen können. Ein drittes Problem sind die verklärenden Stellungnahmen zu historischen Formationen, die längst nicht mehr als so ungebrochen positiv aufgefasst werden, wie dies vor einem Jahrhundert noch für die Epoche der europäischen Aufklärung der Fall war.

Aus dieser Nichtrezeption schwerwiegender Einwände aus feministischer, postund dekolonialer Sicht kann deshalb leicht eine generelle Ablehnung von Stieglers Sprecherposition erwachsen. Wer sich von diesen Schwierigkeiten nicht abhalten lässt, wird allerdings mit einem philosophischen Denken vertraut gemacht, das paläoanthropologische Befunde mit avancierten Positionen der Technikphilosophie ebenso zu verbinden weiß wie Detaillektüren kanonisierter Philosophen (Kant, Husserl, Heidegger) mit tagesaktuellen Marketingbotschaften aus dem digitalen Kapitalismus oder mit Dokumenten bezüglich des von Stiegler mit Horror beobachteten Aufstiegs der Neuen Rechten. ${ }^{2}$ Die Verklammerung all dieser doch sehr divergenten Quellen gelingt dank Stieglers konsequentem Einholen der von ihm konstatierten Technik- und Medienvergessenheit des westlichen Denkens. Als Grundfigur dient ihm dabei die ,tertiäre Retention“, ein Begriff, der an Husserls Theorie der primären und sekundären Retention (Erinnerung) in der Konstitution eines Wahrnehmungsaktes angelehnt ist (Stiegler 2009c, S. 71-80), und den Stiegler mit seiner Theorie des exteriorisierten Gedächtnisses verknüpft (ebd., S. 51-59). Kurz gefasst zeigt Stiegler, inwiefern schon Wahrnehmungen als vermeintlich simple psychische Prozesse von der Mitwirkung exteriorisierter Gedächtnisträger abhängen. Gemeint sind damit alle menschlichen Artefakte, vom Feuerstein bis zum Cloud Computing: Sie alle gelten als tertiäre Retentionen, die jede für sich an der Konstitution psychischer wie sozialer Fähigkeiten ihren Anteil haben. Das Technische und das Menschliche sind also immer schon miteinander verwoben und evolvieren in einer Verflechtung, die es zu analysieren gilt, will man Anthropologie oder Gesellschaftstheorie betreiben.

Bei einer nur kursorischen Rezeption kann es den Anschein haben, als schriebe Stiegler eine klassische Verfallsgeschichte fort, wie sie aus den konservativen Technikphilosophien deutscher und französischer Provenienz bekannt ist. Tatsächlich schiebt Stiegler die Schuld für die katastrophale Lage der Gegenwart (Anthropozän, Klimakrise, Bildungsmisere, Rechtspopulismus etc.) aber keineswegs den Technologien als solchen zu. Vielmehr sieht er die kapitalistische Entwicklung und Implementierung dieser Technologien als das Hauptproblem an bzw. die Konstellation des gegenwärtigen (Finanz-)Kapitalismus mit seinen seit einem Jahrhundert perfektionierten Marketingmechanismen. Demgemäß wird als sein wichtigster politischer Begriff die ,generalisierte Proletarisierung“ gewertet (Ross 2018, S. 21). Man kann

\footnotetext{
${ }^{2}$ Für einen Überblick über Stieglers Philosophie siehe Abbinnett (2017) sowie den von Erich Hörl eingeleiteten und kommentierten Interviewband zwischen Stiegler und Élie During (Stiegler 2009c).
} 
darin den Versuch sehen, einen zentralen Befund von Marx in die Zeit der Massenmedien und der digitalen Medien fortzuschreiben: Das Proletariat markiere nämlich, so Stiegler, nicht so sehr eine Klasse als eine Subjektposition, die durch den Verlust von handwerklichem Können und Herstellungswissen (savoir-faire) bestimmt sei (Stiegler 2010a, S. 66). Was Marx aber nicht voraussehen konnte, sei, dass der Kapitalismus im 20. Jahrhundert durch die Methoden des Marketing eine zweite Phase dieser Proletarisierung ins Werk setzen würde, die ihm zufolge in der Externalisierung nicht nur des savoir-faire der Produzent*innen, sondern auch des savoir-vivre der Konsument*innen besteht (Stiegler 2011, S. 102 ff.). Die generalisierte Proletarisierung wirkt sich nun also nicht nur als habituelle, sondern auch als kognitive und affektive Proletarisierung aus (Stiegler 2010b, S. 30). Nicht überraschen wird, dass Stiegler mit den digitalen Medien der ,hyperindustriellen Gesellschaften“ noch einmal eine neue Phase angebrochen sieht, nämlich in Gestalt einer ,Proletarisierung der noetischen Fähigkeiten zur Theoretisierung und in diesem Sinne zur wissenschaftlichen, moralischen, ästhetischen und politischen Überlegung und Beratung“ (Stiegler 2020, S. 314; Hervorhebung i. O.).

Ein zentrales Werkzeug für diese Gegenwartsdiagnostik ist der Begriffskomplex Psychomacht, Psychotechnologie und Psychopolitik. Dass jede neue Technik das psychische und soziale Gefüge rekonfiguriert, in dem sie sich verbreitet, ist für Stiegler aufgrund der Prämisse von der tertiären Retention unvermeidlich. Insofern kommt jeder Technik auch eine psychotechnische Seite zu - als Psychotechniken im ausdrücklichen Wortsinn werden dann aber von Stiegler doch in erster Linie die Schrift und das Buch angeführt (Stiegler 2008, S. 34). Auch die vom späten Foucault analysierten Techniken des Selbst werden als Psychotechniken in diesem Sinn adressiert (Stiegler 2009b, S. 22). ${ }^{3}$ Dass der Begriff noch sehr viel weiter gefasst werden kann, deutet Stiegler zumindest an, wenn er auch Körpertechniken wie den Tanz, Gymnastik, Sport oder Wandern als Psychotechniken bezeichnet, insofern auch sie Aufmerksamkeit trainieren (Stiegler 2008, S. 61).

Diese Erläuterungen verdeutlichen bereits, dass das psychische Vermögen der Aufmerksamkeit im Zentrum von Stieglers Überlegungen steht, ja dass er das Psychische auf die Aufmerksamkeit sogar zu reduzieren scheint, wann immer er von Psychotechniken spricht - wir werden sehen, ob es bei diesem Befund bleibt. Der Fokus auf die Aufmerksamkeit betrifft jedenfalls besonders Stieglers Abrechnung mit der Kulturindustrie bzw. den von ihm sogenannten „Programmindustrien“, also den ,im audiovisuellen Bereich tätigen Unternehmen“ (Stiegler 2009b, S. 152) heute würde man metonymisch von Netflix als idealtypischem Medienunternehmen sprechen, das die Diagnose einer automatisierten Aufmerksamkeitsbannung in einer gegenüber Radio und Fernsehen nochmal verschärften Form plausibilisiert. Die Automatisierung verweist auf die Verschiebung von den schon für die Antike (wenn nicht für die gesamte Menschheitsgeschichte) aufweisbaren Psychotechniken zu den Psychotechnologien, die auf industriellen Produktionsweisen und wissenschaftlicher Forschung beruhen und deren Funktionieren nicht mehr von habituellen Praktiken

\footnotetext{
3 Foucault wird dafür gewürdigt, ,die erste Genealogie der Psychotechniken“ vorgelegt zu haben: „Was Foucault in diesem Rahmen als Archäologien bezeichnet, sind für uns retentionelle Dispositive" (Stiegler 2008, S. 142).
} 
von Subjekten abhängt. Vielmehr sind Psychotechnologien in Geräte eingeschrieben, die die nunmehr zumindest potenziell passivierten Subjekte unaufhörlich bespielen und kontrollieren. Allerdings schreibt Stiegler den Willen zur ,psychischen Kontrolle über die Bevölkerung" nicht mehr dem Staat zu, sondern den Unternehmen, also kapitalistischen Akteur*innen (Stiegler 2009b, S. 163). Stiegler greift zudem auf Deleuzes (1993a) Begriff der Kontrollgesellschaft zurück, um diese Verschiebung zu verdeutlichen. Die Stichworte „Kontrolle“, „Modulation“ und „Marketing“ erlauben Stiegler die Fortschreibung der Foucaultianischen Genealogie der Macht, wenn er konstatiert, dass es ,,jenseits der Biomacht eine Psychomacht gibt, eine Macht also, die über die Seelen ausgeübt wird“ (Stiegler 2009b, S. 56f.). ${ }^{4}$ Diese Psychomacht wird demzufolge als eine globale, systematische Vereinnahmung der Aufmerksamkeit definiert, die durch die Psychotechnologien Radio, Fernsehen und digitale Medien ermöglicht wurde (Stiegler 2013, S. 81).

Aber geht Stiegler damit über die altbekannten Klagen von Kritiker*innen der Kulturindustrie oder der Aufmerksamkeitsökonomie überhaupt hinaus? Was die Begriffsprägung Psychomacht motiviert, ist erst zu erfassen, wenn Stieglers Überzeugung in den Blick rückt, dass die Medienkonsument*innen durch die Vereinnahmung der Aufmerksamkeit gerade nicht nur in einer immer gleichförmigen Gegenwart festgesetzt werden, sondern dass damit zugleich ihr Bezug zur Zukunft beschnitten wird. Die wichtigste, aber offenbar auch prekärste psychische Funktion ist ihm zufolge das Vermögen, sich auf nicht-existierende Objekte zu beziehen - auf das, was er als Konsistenzen bezeichnet und als unendliche Gegenstände fernab jeder Berechenbarkeit charakterisiert. ${ }^{5}$ Man könnte darin Glaubensinhalte jeglicher Art verstehen. In der Philosophie sind das klassischerweise Werte und Ideen, und zwar nicht nur im platonischen Sinn; auch Kant und die ihm folgende kritische Tradition hält etwa an regulativen Ideen fest, und selbst die Dekonstruktion kennt Begriffe wie Gabe oder Gerechtigkeit, die sich durch empirische, kalkulierbare Verhältnisse niemals ausschöpfen lassen. Für Derrida eröffnen nichtempirische Begriffe wie diese in eminenter Weise den Horizont eines unendlichen Kommens im Sinne einer radikal offenen Zukunft. Stiegler schließt sich diesem Theorem an, fundiert es allerdings im Begehrensvermögen der Psyche, das immer schon von der Technik mitkonstituiert ist. Er scheut sich auch nicht, den Glauben an Konsistenzen (als das, was nicht existiert) als eine Projektion zu bezeichnen, stellt aber sogleich klar, dass diese Projektion irreduzibel für ein ,nicht-unmenschliches“ Leben ist (vgl. Stiegler 2019, S. 197).

Die Aufmerksamkeit ist also nicht das einzige psychische Vermögen, das Stiegler im Sinn hat, wenn er von Psychotechniken, Psychotechnologien oder Psychomacht spricht. Systematisch gesehen nimmt das Begehren und die für das Begehren konstitutive Fähigkeit zur Projektion (man könnte auch von Imagination oder ganz

\footnotetext{
4 Auch hier ist Foucault inhaltlich für die Beschreibung des Pastorats als einer Macht über die Seelen der Einzelnen zu würdigen; Stiegler geht aber terminologisch einen Schritt weiter.

5 „Die Konsistenz des Konsistenten liegt auf einer anderen Ebene als derjenigen der Existenz, denn sie kann nicht bewiesen und noch weniger kalkuliert werden. [...] Eine Konsistenz ist ein Objekt der Sublimierung. Ein solches Objekt ist inexistent, wenn es wahr ist, dass nur das existiert, was sich im Raum und der Zeit befindet und insofern berechenbar ist." (Stiegler 2010a, S. 40).
} 
klassisch von der Einbildungskraft sprechen) bei ihm die zentrale Stelle im psychischen Apparat ein. Denn nur sie erlaubt diesem Apparat, erfinderisch zu werden, indem sie Konsistenzen projiziert und damit ein unendliches Begehren motiviert, das in seinem Streben nach Erfüllung kreative Sublimationen hervorbringt, die das Leben des Geistes ausmachen.

\section{Psychopolitik: Variationen eines Begriffs}

Es lohnt sich nun, Stieglers Terminologie mit ähnlichen Vorschlägen zur Einführung angemessener Begriffe für die gegenwärtigen Machtverhältnisse zu vergleichen. Im deutschen Sprachraum bieten sich dafür gleich zwei Arbeiten an, die beide das Wort Psychopolitik im Titel tragen: die Monographie der Soziologin Alexandra Rau (2010) sowie der Essay des Philosophen Byung-Chul Han (2014).

Rau folgt wie schon Stiegler den Ansätzen Foucaults rund um die Theorie der Biomacht und der Gouvernementalität, verfolgt dabei aber keinen technikphilosophischen, sondern einen gesellschaftstheoretischen Ansatz, der nach den Verschiebungen in den Weisen der Subjektivierung fragt. Gleichwohl schlägt auch sie den Terminus Psychopolitik vor, der ihr zufolge ,eine jüngere gesellschaftliche Machtform ist, durch die Menschen ,geführt" werden und ,sich selbst führen “" (Rau 2010, S. 15). ${ }^{6}$ Die Begriffswahl geht auf die seit den 1980er-Jahren vielfach analysierte „Psychiatrisierung des Alltags“ zurück (Castel Castel et al. 1982), die auch das Berufsleben zunehmend transformiert. Die Pointe in Raus Anknüpfung an diese Arbeiten liegt darin, dass diese Psychiatrisierung auch ,eine neuartige Form des Subjekts“ (Rau 2010, S. 280) generiert hat, die auf ,psycho-logischem Wissen“ beruht und letztlich Individualität im modernen Sinn ,,produziert“ (vgl. Sonntag 1988). Wie schon Stiegler verknüpft auch Rau diese individualitätsgeschichtliche Verschiebung mit Deleuzes Diagnose des Entstehens der Kontrollgesellschaften und liest sie als deren charakteristische Regierungsweise (Rau 2010, S. 298 ff.). Zwar spricht Rau nicht von Psychomacht, sondern nutzt der expliziten Anknüpfung an Foucault zum Trotz den Begriff der Psychopolitik. Allerdings scheint sie im Grunde dieselbe Geschichte eines zunehmend invasiven und tatsächlich herrschaftsförmigen Machtverhältnisses zu beschreiben, wie Stiegler in seiner Darstellung der Industrialisierung und folglich Proletarisierung aller Lebensbereiche.

Rau ist allerdings die konsequentere Foucault-Leserin, wenn sie diese Darstellung einer zunehmenden Unterdrückung immer wieder durch einen zweiten Vektor konterkariert: „Es wäre nun allerdings komplett falsch“, schreibt sie, ,die Erzählung des Aufstiegs des ,Psycho-Logischen“, wie bisher, in der Tendenz einer erfolgreichen Herrschaftsgeschichte zu belassen“ (ebd., S. 282). Die Entschiedenheit der Wortwahl sticht in einer sonst sehr vorsichtig formulierten Arbeit heraus. Rau verdeutlicht, inwiefern gerade die Produktion eines an sich bzw. an seiner Psyche arbeitenden Subjekts auch dessen Widerständigkeit mobilisiert, etwa in Form von „Gegenregierungen“ gegen konkrete Institutionen, die die Psychologisierung forcie-

\footnotetext{
6 Anders als Stiegler geht Rau in einem aufschlussreichen Kapitel auf die Geschichte der industriellen Psychotechnik im frühen 20. Jahrhundert ein (Rau 2010, S. 247-267).
} 
ren, oder sogar gegen herrschaftliche Regierungsformen überhaupt, wie Rau unter Rekurs auf die Rezeptionsgeschichte der Kritischen Theorie etwa Marcuses zeigt (vgl. S. 282-286). Die Heraufkunft der Psychopolitik als einer neuen Machtform ist folglich nicht nur als eine Geschichte der Unterwerfung, sondern auch der Ermächtigung zu erzählen - und als eine Geschichte neuer und intensivierter Kämpfe (vgl. S. 417), nämlich in Gestalt eines positiven Kampfes um Selbstentfaltung sowie eines negativen um die „Verteidigung des Selbst“ (S. 420). Die Frage, mit der Rau endet, ist eine eminent politische: Wie lassen sich diese individuell geführten ,psycho-logischen“ Kämpfe in kollektive Kämpfe überführen und damit politisieren? (S. 422).

Der Begriff der Psychopolitik bringt diese Ambivalenz klarer zum Ausdruck als die Psychomacht, von der Stiegler spricht. Denn in seiner Charakterisierung der Psychomacht versäumt er es, die wichtige Unterscheidung zwischen Macht und Herrschaft aufzugreifen, auf die der späte Foucault gerade in Absetzung gegenüber dem Zusammenfallen beider Begriffe gepocht hat, wie ihm dies in seinen früheren, machtkritischen Arbeiten unterlaufen sei (Foucault 2005, S. 900). Macht, so Foucault, ist produktiv, sie ermöglicht und erzeugt etwas, und sie tritt immer nur in einem differenziellen und dynamischen Kräfteverhältnis in Erscheinung. Herrschaft ist demgegenüber ein stabilisierter Machtvektor, der diese Dynamik temporär stillstellt. Wenn Stiegler von Psychomacht spricht, ist immer ein Herrschaftsverhältnis gemeint, sodass er eigentlich von Psychoherrschaft sprechen müsste - ein schrecklich klingender Begriff, der angesichts der Charakterisierung Stieglers, der zufolge die Psychomacht eine Ausübung von Macht über die Seelen sei (Stiegler 2009b, S. 56f.), aber durchaus angemessen wäre. Nun gebraucht zwar auch Stiegler den Ausdruck Psychopolitik, allerdings nur im Sinn einer Forderung nach einem Ausweg aus der von ihm diagnostizierten Beherrschung durch die Psychomacht. Psychopolitik ist bei ihm das Desiderat, nicht eine bereits existierende Instanz. Anders gesagt, denkt Stiegler Psychopolitik als solche gar nicht; zumindest entwickelt er von ihr, ganz im Gegensatz zu Rau, keinen Begriff.

Auch der Philosoph Byung-Chul Han knüpft in seinem Essay Psychopolitik an Foucault und Deleuze an und spitzt die nun bereits bekannte Motivik zu: Foucault bleibe zu sehr an den Überlegungen zur Biopolitik hängen und verpasse damit die Chance, das neoliberale Regime zu verstehen (Han 2014, S. 37), wohingegen Deleuze im Essay zu den Kontrollgesellschaften die Psychopolitik der Sache nach durchaus beschreibe, wenngleich nicht so benenne (ebd., S. 30). Für Han jedenfalls ist ausgemacht, dass die Psychopolitik die Regierungsform des neoliberalen Regimes ist und mehr noch, dass der Neoliberalismus die Psyche als Produktivkraft überhaupt entdeckt hat (ebd., S. 39). In dieser Zuspitzung ist letzteres sicherlich falsch. Sowohl Illouz (2007) als auch Rau (2010) weisen unter Rückgriff auf die Kulturgeschichte der Psychodisziplinen nach, dass die Etablierung der Psyche als eines Produktivfaktors im Kapitalismus in die erste Hälfte des 20. Jahrhunderts zurückreicht.

Han ist ein Meister der Zuspitzung und der Polemik, aber nicht unbedingt der Differenzierung. So wird Rau in einer Fußnote einerseits erwähnt, aber sofort wieder liegengelassen, weil ihr Begriff der Psychopolitik noch in biopolitischen Kategorien verharre (Han 2014, S. 116). Eine differenzierte Betrachtungsweise zeigt hingegen, 
dass Rau durchaus das Unzureichende eines bloß biopolitischen Begriffsapparats für die Analyse gegenwärtiger Verhältnisse der Macht und Subjektivierung herausarbeitet. Im definitorisch klaren Gebrauch des Begriffs Psychopolitik geht ihre Arbeit dem Essay Hans einige Jahre voraus. Ähnlich geht Han mit Stiegler vor: Dem französischen Philosophen wird zugestanden, einen angemessenen neuen Machtbegriff eingeführt zu haben, nämlich eben die Psychomacht. Doch zugleich habe Stiegler die älteren Medien des Buchs und des Fernsehens überbetont und die ,genuin digitalen Medien wie Internet und Soziale Medien und deren Kommunikationsstruktur" nicht ausreichend beachtet: „Dadurch verfehlt er die neoliberale Psychopolitik völlig, die sich massiv der digitalen Technik bedient" (ebd., S. 41). ${ }^{7}$

Allerdings kommt Han zu denselben drastischen Aussagen, wenn er etwa konstatiert, dass die neoliberale Psychopolitik mit ihrer Bewusstseinsindustrie die menschliche Seele ,zerstört“ (S. 46). Weiterführend ist allerdings die Beschreibung einer Gewalt, die vom „Diktat der Positivität“ ausgehe, mit der die digitalen Medien operieren (S. 45). Die neue Machttechnik operiere mit positiven Anreizen (likes), schmeichle damit der Seele und verführe sie so letztlich wirksam und zuverlässig: „Die neoliberale Psychopolitik ist eine Smarte Politik, die zu gefallen, zu erfüllen sucht, statt zu unterdrücken“ (S. 51). Anders gesagt, sie bemächtigt sich der Emotion auf einer ,,präreflexiven Ebene“ und beeinflusst dadurch Verhalten und Handeln der Individuen (S. 67). Han spricht den um 2014 noch jungen Diskurs über das MicroTargeting an, also die Beeinflussung einzelner Wähler*innen mit personalisierten Botschaften, und bezeichnet dies als ,datengetriebene Psychopolitik“, die imstande sei, ,in die psychischen Prozesse prospektiv einzugreifen“ (S. 86). Das aber würde die Umgehung oder Unterwanderung des Willens und damit das Ende der Freiheit bedeuten. Zusammen mit Big Data würde so das „Kollektiv-Unbewusste“ einer ganzen Gesellschaft entschlüssel- und manipulierbar: „Die digitale Psychopolitik wäre dann in der Lage, sich des Verhaltens der Massen auf einer Ebene zu bemächtigen, die sich dem Bewusstsein entzieht" (S. 89). Am Ende liefert Han eine Definition der neoliberalen Psychopolitik als einer „Herrschaftstechnik, die vermittels psychologischer Programmierung und Steuerung das herrschende System stabilisiert und fortführt“" (104f.).

Der Gegenwartsdiagnose einer Heraufkunft der Psychomacht bei Stiegler fügen somit sowohl Rau als auch Han wesentliche Aspekte hinzu: Rau ist als konsequente Foucault-Leserin hellhörig für die Gleichursprünglichkeit von Herrschaftssowie von Widerstandsvektoren innerhalb eines Machtgefüges, auch wenn es sich um ein neues Feld handelt, auf dem sich diese Kämpfe abspielen, wie es mit der Psychopolitik für sie ganz klar der Fall ist. Zugleich fehlt in ihrer Darstellung die Auseinandersetzung mit der Rolle der Medien in diesen Kämpfen. Han wiederum hat darin Recht, dass Stiegler bei seiner Diskussion der Psychomacht zu wenig auf die Rolle der digitalen Medien eingeht, die gegenüber den klassischen Massenmedien

\footnotetext{
7 Dieser Eindruck ist, wenn man nur die bis ca. 2010 vorliegenden Publikationen Stieglers liest, nachvollziehbar. In den 2010er Jahren wurde allerdings erkennbar, dass Stiegler sich den „,genuin digitalen Medien“ zunehmend zugewandt hat und diese sich in seine Analysen gut eingliedern ließen, ohne dass das generelle Framework hätte geändert werden müssen - siehe etwa seine Hinweise zur „digitalen Pharmakologie“ (Stiegler 2016).
} 
die Individuen auf eine andere und neuartige Weise adressieren, nämlich personalisiert, automatisiert, responsiv und damit zunehmend verführerisch. Um den neuen Schauplatz dieser Machtkämpfe - nämlich die Psyche - angemessen ausflaggen zu können, braucht es daher ein klareres Bewusstsein darüber, welche psychischen Vermögen bereits heute durch digitale Technologien adressiert und manipuliert werden können, bzw. mit welchen Zugriffsmöglichkeiten in naher Zukunft gerechnet werden muss. Es braucht, mit anderen Worten, einen Überblick über das psychotechnologische Arsenal der Gegenwart. Um einen Satz von Max Horkheimer zu paraphrasieren sowie eine berühmt gewordene Wendung Dwight Eisenhowers aus seiner Abschiedsrede als US-Präsident abzuwandeln: Wer von Psychopolitik sprechen will, darf vom psychotechnologischen Komplex nicht schweigen - denn nur so kann auch ein Diskurs über den Schutz vor dem unbefugten Einfluss geführt werden, der von diesem Komplex gewollt oder ungewollt ausgeübt wird.

\section{Das psychotechnologische Arsenal}

Stieglers zeitdiagnostischer Psychomacht-Befund stützt sich im Wesentlichen auf die Annahme, dass private, gewinnorientierte Medienkonzerne tatsächlich in der Lage sind, die Aufmerksamkeitsströme ihres Publikums zu lenken. Die Klage über das, was die Kritische Theorie Kulturindustrie, Vergnügungsindustrie oder Bewusstseinsindustrie genannt hat und was seit den späten 1990er-Jahren unter Aufmerksamkeitsökonomie neu verhandelt wurde, ist zwar altbekannt, aber das ist noch kein Grund, sie als überholt aufzufassen. Die Etablierung zunächst des Internets, dann der Sozialen Medien, der Smartphones und der Streamingdienste hat gezeigt, dass damit jedes Mal ein neues Aufmerksamkeitsregime eingeführt wurde, dessen disruptive Wirkung nicht ignoriert werden kann. Weniger bekannt ist, wie sehr die IT-Industrie die Fesselung der Aufmerksamkeit von Anfang an das Design ihrer Produkte hineinprogrammiert. So ist etwa Bingewatching kein Betriebsunfall der Ausbreitung von Streamingdiensten, sondern deren innerste Absicht (vgl. Zündel 2017, S. 32).

Im Folgenden soll ein paradigmatischer Design-Ansatz für IT-Anwendungen diskutiert werden, um zu zeigen, dass die Psychotechnologie sich keineswegs nur auf die Erregung und Kontrolle von Aufmerksamkeit reduzieren lässt, sondern dass mehrere weitere psychische Ebenen adressiert werden: Überzeugungen, Einstellungen, Affekte sowie mit all dem verquickt das Verhalten. Auf dieser Grundlage wird dann die Frage diskutiert, inwiefern die IT-Industrie zur Rekonfiguration der Psyche beiträgt, ja mehr noch: inwieweit sie die Psyche gerade von digital natives überhaupt mitkonstituiert. Wie eng das Design von Computerinterfaces und der Funktionalität der Anwendungen mit verhaltenspsychologischen Forschungen verzahnt ist, lässt sich anhand des Fallbeispiels der Persuasive Technology zeigen. Unter diesem auf die Rhetoriktradition anspielenden Buchtitel beschäftigt sich der in Stanford lehrende Verhaltenspsychologe B. J. Fogg mit der Frage, wie sich Computer zur 
Veränderung von Denken und Verhalten einsetzen lassen. ${ }^{8}$ Fogg bemüht sich zwar darum, den Begriff der Persuasion von dem des Zwanges (coercion) und dem der Täuschung (deception) abzugrenzen: Zwang sei mit Gewalt (force) verbunden, wogegen Persuasion freiwillige Änderung (voluntary change) impliziere (Fogg 2003, S. 15). Allerdings räumt Fogg selber ein, dass die Grenze zwischen Persuasion und Zwang eine sehr feine sei. Fogg beschreibt Nutzer*Innen so, als stünden sie ihren Medienumgebungen immer als vollbewusste, autonome Subjekte gegenüber, die jederzeit in der Lage sind zu entscheiden, ob sie einem Persuasionsversuch stattgeben wollen oder nicht. Aber wie soll man sich das vorstellen? Denn auf das Auftreten von Persuasionsversuchen durch digitale Technologien haben Nutzer*innen, sofern sie kein anti-digitales Leben führen, ja keinen Einfluss. Sie können immer erst reaktiv entscheiden, ob sie dem Versuch einer Verhaltens- und Gedankenmodifikation stattgeben wollen oder nicht. Zu dieser Entscheidung werden sie aber genötigt, sobald Persuasionstechnologien implementiert sind. ${ }^{9}$

Diesen Aspekt der Nötigung scheint auch das Kunstwort zum Ausdruck zu bringen, das Fogg eingeführt hat: captology, abgeleitet von computers as persuasive technologies. Die Konnotation von capture, also Gefangennahme und Einkerkerung, wird von Fogg nicht reflektiert. Darauf weist der Computerspielforscher Ian Bogost hin, der seinerseits die Persuasion zum Grundbegriff der von ihm so genannten Persuasive Games gewählt hat, und der in seiner Auseinandersetzung mit Fogg zu dem Schluss kommt, dass der treffendere Begriff ,manipulative technology“ sei, weil sie nicht darauf abgestellt sei, einen politischen Diskurs zu eröffnen, wie das für gute Rhetorik gelte (Bogost 2007, S. 62). ${ }^{10}$ Zehn Jahre nach dieser Einschätzung wurde durch eine Reihe von Reportagen in The Guardian sowie einigen vielbeachteten TedTalks mit Sorge diskutiert, wie weit sich die manipulative Kraft des Interfacedesigns bereits entwickelt hatte. Ehemalige Silicon-Valley-Entwickler*innen begannen vor ihren eigenen Produkten zu warnen: Ein Mitarbeiter von Fogg verglich die Methoden der Einflussnahme, die im Stanforder Persuasive Technology Lab mitentwickelt wurden, mit den perfekt auf die menschliche Psyche abgestimmten Mechanismen von Spielautomaten (Lewis 2017); ein Vergleich, der von der Ethnologin Natasha Dow Schüll, die über die Spielautomatenkultur in Las Vegas geforscht hat, bestätigt wurde (Busby 2018). Tatsächlich findet man Buchtitel wie den Bestseller Hooked. How to Build Habit-Forming Products (Eyal 2014), die keinen Zweifel daran lassen,

\footnotetext{
8 In seinem Vorwort zu Foggs Buch bemüht sich der mit den Stanford Prison Experiments berühmt gewordene Philipp Zimbardo sichtlich, auf die Gefahren der persuasive technologies hinzuweisen und das Wissen um ihre Macht zum Schutz vor ihrem ungewünschten Einsatz durch Dritte stark zu machen: „The time has come for us to pay attention to how technology can — and will—be designed to change how people think and what they do. Not to do so would be at our peril. We would miss opportunities to bring about positive, desired changes in people's lives. We would be slow to take necessary steps against unwanted persuasion by unethical agencies using this new technology for profit or political advantage." (Fogg 2003, S. xii).

9 Ähnlich argumentiert Distelmeyer mit Bezug auf die „Ästhetik der Verfügung“ (Distelmeyer 2017) von Interfaces beliebter Internet-Plattformen wie YouTube.

10 Die oberflächliche Übernahme der rhetorischen Kategorie der Überzeugung bei Fogg erweist sich letztlich als zynisch, wenn es keine Rede mehr gibt, die an jemanden adressiert wird und die gehört und kognitiv wie affektiv verarbeitet wird.
} 
dass zumindest Marketing-Expert*innen die technologische Tendenz zur Heteronomisierung von Interface-Nutzer*innen rückhaltlos auszubeuten bereit sind.

Um eine andere, individuell zugeschnittene Art von Ausbeutung geht es beim (schon von Han erwähnten) Micro-Targeting, einer Methode der Werbung, die ihre Botschaften nicht mehr wahllos an alle richtet, sondern stattdessen immer feinkörniger gerasterte Zielgruppen definiert und adressiert. Micro-Targeting beruht auf digitaler Psychometrie, also der Vermessung psychischer Eigenschaften, die zur Erstellung von Persönlichkeitsprofilen dient. Diese ,algorithmische Psychometrie“ knüpft gewissermaßen da an, wo Francis Galton im späten 19. Jahrhundert angesetzt hat, wie der STS-Forscher Luke Stark verdeutlicht. Galton definierte die Psychometrie als ,the art of imposing measurement and number upon operations of the mind“ (Stark 2018, S. 209); eine Definition, die auch für die zeitgenössischen Anwendungen gültig bleibt. Die digitale Psychometrie soll anhand der Auswertung von Datenbeständen eine feinkörnige Persönlichkeitsmodellierung ermöglichen. Daraus ergibt sich der Reiz, den diese algorithmische Psychometrie für digitales Micro-Targeting hat. Es wird aber zugleich sichtbar, dass mit diesen neuen Technologien, für deren politische Dimension die Techniksoziologin Zeynep Tufekci den Begriff einer „computational politics“ vorgeschlagen hat, das Wesen der politischen Öffentlichkeit fundamental unterlaufen wird, denn: ,Computational politics turns political communication into an increasingly personalized, private transaction and thus fundamentally reshapes the public sphere" (Tufekci 2014, S. 2). Die Zerschlagung der Sphäre der Öffentlichkeit in unzählige Mikro-Öffentlichkeiten unterläuft die Möglichkeit, an einer gemeinsam geteilten Realität teilzunehmen. Tufekci, Stark und andere kritische Forscher*innen der Verschränkung von Psychologie und Computerwissenschaft fordern daher zu Recht auf Emanzipation und Wohlergehen ausgerichtete, progressive computational politics, die auf die antidemokratische Herausforderung durch den „psycho-computational complex“ reagieren (Stark 2018, S. 221). ${ }^{11}$

Während Persuasionstechnologien und Micro-Targeting ihren Akzent auf die Beeinflussung von Aufmerksamkeit, Meinungsbildung und Überzeugungen legen, lässt sich seit einigen Jahren ein Trend zur gezielten Adressierung von Stimmungen, Affekten und Emotionen feststellen. Technisch relativ simpel operieren hierbei Anwendungen zum „Mood Tracking“ (Pritz 2016) sowie Wellness- und Meditations-Apps, die zunehmend zur individuellen Stimmungsregulation herangezogen werden. Die Nutzer*innen müssen hierbei an der Aufzeichnung ihrer Stimmungen meist noch aktiv mitwirken sowie Aufgaben wie zielgerichtete Meditationen zur Hebung ihrer Laune absolvieren. Achtsamkeit wird dabei aus den vordigitalen Praktiken dekontextualisiert und einer kalkulierten Indienstnahme zugeführt, die nicht nur klare Vorgaben für die erwarteten Zielzustände (happiness) macht, sondern diese zudem mit Produktivität und Konkurrenzfähigkeit verknüpft, was aus Sicht kritischer Psycho$\log *$ innen perfekt in die neoliberale Subjektanrufung passt (Slunecko und Chlouba 2021).

Einen entscheidenden Schritt weiter gehen automatisierte Affekttechnologien, die, konsequent weitergedacht, zu einer blasenförmigen Selbstabschließung von Nut-

${ }^{11}$ Starks Begriffsschöpfung ,psycho-computational complex“ hat den Titel des vorliegenden Aufsatzes mitinspiriert. 
zer*in und digitaler Umgebung, etwa in Form von „Empathie-Maschinen“ (Turkle 2019), führen könnten. Unter den Bezeichnungen affective computing (Picard 1997), affective media (Bösel und Wiemer 2020), empathic media und emotional AI (McStay 2018) wird die digitale Vermessung von Affekten und Emotionen ebenso adressiert wie die Simulation von Emotionen durch künstliche Gefährt*innen sowie das Management des emotionalen Lebens von Nutzer*innen. Tendenziell wird mit diesen Innovationen also das Vermögen zur Affekt- und Emotionsregulation ausgelagert: Auf Basis der Datafizierung von Körperparametern (allen voran die Gesichtsmimik) sowie von Verhaltensmustern unterbreiten Algorithmen Vorschläge zur Verbesserung des subjektiven Wohlbefindens oder simulieren die Präsenz eines empathischen Gegenübers. Abgesehen von der Datenschutzproblematik, die bezüglich affektiver Daten nochmals potenziert erscheint, ist die Implementierung dieser Technologien schon aufgrund der dabei verwendeten, höchst umstrittenen Theorien angeblich universeller Basisemotionen fragwürdig (Feldman Barrett 2017). Die Kategorisierung des psychischen Lebens beruht auf überaus simplifizierenden Modellen und Kategorienfehlern (vgl. Weigel 2020). Gleichwohl werden so neue Realitäten geschaffen, weil Nutzer*innen zumeist nicht wissen, nach welchen Prinzipien hier überhaupt vorgegangen wird, was einem ,blackboxing“ des psychischen Lebens gleichkommt (Bösel 2020). Marktreife Anwendungen wurden zunächst für den Marketing-, den Gesundheits-, den Bildungssektor und natürlich die Unterhaltungsindustrie entwickelt. Als langfristiges Ziel werden automatisierte Agent*innensysteme sowie soziale Roboter imaginiert, die auf ihre Nutzer*innen so perfekt abgestimmt sind, dass die emotionalen Bedürfnisse hinreichend gedeckt werden, was Stieglers Verdacht plausibel erscheinen lässt, dass diese Technologien langfristig zur affektiven Proletarisierung führen könnten - im Sinne eines Verlusts an Wissen zur individuellen Affekt- und Emotionsregulation.

Darüber hinaus wird inzwischen deutlich, wie sehr sich Anwendungen, die ein verdatetes Wissen um die emotionalen und psychischen Zustände beobachteter Personen versprechen, in die Arbeit von Exekutivbehörden eingliedern lassen. So verspricht man sich etwa vom Projekt iBorderCtrl die Erkennung unterstellter Täuschungsabsichten von Migrant*innen. Das Politikum „Grenzschutz“ wird hier mit der Implementierung pseudowissenschaftlicher Methoden der emotion detection noch weiter aufgerüstet, denn: „The desire to find a ,scientific ' method to detect lying, affective states and emotions is what drives the investment in deception detection and emotional AI more broadly. This desire or political will far outpaces the validity of the technology." (Sánchez-Monedero und Dencik 2019, S. 6). Wenn dem so ist, reduziert sich der politische Wille allerdings auf technologischen Solutionismus, also den Versuch, soziale Probleme durch (noch dazu unausgereifte) algorithmische Automatisierungen zu lösen, bevor sie überhaupt einer adäquaten Analyse unterzogen wurden (vgl. Morozov 2013). 


\section{Die Rückkehr des Behaviorismus und der psychotechnologische Paternalismus}

Die psychotechnologische Beeinflussung von Aufmerksamkeit, Überzeugungen und Affekten kulminiert darin, dass das Verhalten von Nutzer*innen digitaler Umgebungen zunehmend anhand undurchsichtiger Kriterien kontrolliert und gesteuert wird. Kritische Theoretiker*innen konstatieren seit einigen Jahren die Rückkehr behavioristischer Programme auf der Basis digitaler Datenpraktiken - die Rechtsphilosophin Antoinette Rouvroy prägte dafür den Begriff ,data behaviorism“ und interpretiert diesen als Kernstück der ,algorithmischen Gouvernementalität“ (Rouvroy 2013), die über das Prozessieren von Daten regiert und damit die sprachbasierten Sphären des Juridischen und des Politischen unterläuft. Auch William Davies zieht in The Happiness Industry das Fazit, dass mit dem Aufschwung der Datenwissenschaft insgesamt der behavioristische und utilitaristische Ehrgeiz zum Management der Gesellschaft neu entfacht wurde, was aus Sicht der Demokratie letztlich desaströs sei, weil damit der öffentliche Diskurs um Werte umschifft werde (Davies 2016, S. 222 ff.). Shoshana Zuboff arbeitete schließlich die Parallelen zwischen Behaviorismus und dem von ihr so bezeichneten ,Überwachungskapitalismus“ heraus, indem sie die Forschungsprogramme B. F. Skinners und Alex Pentlands, dem Begründer der datenbasierten „Sozialphysik“ (Pentland 2014), einem ausführlichen Vergleich unterzog. Wie schon der Behaviorismus verspricht sich auch die Sozialphysik einen Qualitätssprung gerade durch die Unterlaufung des menschlichen Bewusstseins (vgl. Zuboff 2018, S. 489) - und zwar mithilfe der Abschöpfung personenbezogener Daten durch Wearables (Nosthoff und Maschewski 2019). Darin trifft sich sie sich mit den aus dem persuasive design geborenen Interfaces, die dann am besten funktionieren, wenn Nutzer*innen nicht merken, nach welchen Prinzipien sie ,überzeugt“ werden. Und ebenso lässt sich für die Entwicklung der affektiven oder empathischen Medien beobachten, dass sich Entwickler*innen lieber auf die vermeintliche Unbestechlichkeit körperlicher Messdaten als auf Selbstaussagen von Nutzer*innen verlassen: ,the conscious mind is not denied, but instead not trusted“ (McStay 2018, S. 60).

Dass so deutliche Kritik an den behavioristischen Aspekten des Daten-, Informations- oder Überwachungskapitalismus ausgerechnet von Wirtschaftswissenschaftler*innen wie Davies oder Zuboff formuliert wird, ist kein Zufall. Gerade in dieser Disziplin fand in den 2000er Jahren ein Paradigmenwechsel statt, der die Konsequenzen aus dem Scheitern des homo oeconomicus-Modells zieht. Die Verhaltensökonomie antwortet auf die Krise der klassischen Ökonomie und ihrer unterkomplexen Anthropologie und wird u. a. durch den Bestseller Nudge von Richard Thaler und Cass Sunstein (2009) weltweit propagiert. Ausgehend von der These, dass Individuen oftmals nicht in der Lage sind, sich für die für sie nutzenbringendste Alternative zu entscheiden, wollen Thaler und Sunstein „kluge Entscheidungen“ in Fragen der Gesundheit, des Wohlstands und des Glücks anstoßen. Für diese und andere Bereiche wird die Etablierung von „Entscheidungsarchitekturen“ angeraten, um Individuen „sanft“ in die angenommene beste Richtung zu stupsen. Mit dem „libertären Paternalismus“ als drittem Weg zwischen konservativer und progressiver Politik forcieren die Autoren (ebd., S. 331 ff.) die Ideologie der Ideologielosigkeit, 
die für den Neoliberalismus so kennzeichnend ist. Auch wenn von persuasiven Technologien in Nudge nicht die Rede ist und die Digitalisierung nicht einmal gestreift wird, dürfte klar sein, dass der libertäre Paternalismus und die digitalen Methoden der Persuasion perfekt zusammenpassen, weshalb die Kombination dieser beiden Ansätze, das „,digital nudging“, auch nicht lange auf sich warten ließ (Weinmann et al. 2016; vgl. dazu kritisch Mühlhoff 2018).

Doch auch am anderen Ende des ideologischen Spektrums hat der Paternalismus längst angefangen, digitale Verdatungsmethoden für seine Belange zu nutzen. Das chinesische Sozialkreditsystem ist derzeit das aufsehenerregendste Beispiel für den autoritären Paternalismus. Im Westen wird dieses System gerne als Umsetzung totalitärer Überwachungsfantasien à la Orwell gedeutet. Ein Blick in die Forschungsliteratur zeigt zwar, dass dieses System zumindest bislang bei weitem nicht so perfekt integriert ist, wie dies oftmals kolportiert wird (Arsène 2019). Dennoch ist zu erwarten, dass die verschiedenen Ansätze einer Vertrauensindizierung von Bürger*innen zunehmend mit weiteren Datensätzen und Methoden wie etwa der automatischen Gesichtserkennung integriert werden; zudem gibt es bereits schwarze Listen von als nicht vertrauenswürdig eingestuften Personen inklusive ihrer öffentlichen Zurschaustellung am digitalen Pranger (Groot 2020). Chinas Bemühungen zur datengestützten verhaltenstechnologischen Sicherstellung sozialer Ordnung sind aber nicht das einzige Projekt dieser Art. Etwa zur selben Zeit wurden Berichte über einen Ableger von Alphabet/Google namens Sidewalk Labs publik, dessen Ziel die Entwicklung smarter Cities und smarter Communities ist, wobei dieses Konzept auch die Verteilung von Privilegien an jene Bewohner*innen vorsieht, die mit ihren Daten besonders freigiebig umgehen. Zuboff sieht diese Vision als Beleg dafür, dass gerade auch westliche Unternehmen die Möglichkeiten und den Willen haben, ,unser Verhalten zu beeinflussen, zu modifizieren und schließlich zu steuern" (Zuboff 2018, S. 266).

Die Konvergenz all dieser Entwicklungen lässt es angemessen erscheinen, von einem psychotechnologischen Paternalismus zu sprechen, der sich gegenüber ideologischen Positionierungen weitgehend gleichgültig verhält. Denn sowohl an den libertären als auch an den autoritären Paternalismus lassen sich Ansätze wie persuasion technologies, das ökonomische und politische Micro-Targeting, die Entwicklung von empathic oder affective media sowie weitere Methoden wie der für nudgingAnwendungen höchst brauchbare gamification-Ansatz (Schrape 2014) nutzbringend andocken. In all diesen Fällen werden Nutzer*innen von ihren digitalen Umgebungen so adressiert oder bespielt, dass das reflexive Bewusstsein weitgehend umgangen werden soll; stimuliert und manipuliert wird hingegen die ,nichtbewusste Kognition“ (Hayles 2017). In die Terminologie der Verhaltensökonomie übersetzt, zielen diese Anwendungen auf das ,schnelle Denken“ ab (Kahneman 2012), das als effizient, aber unzuverlässig gilt, weshalb die Unterstützung durch digital-paternalistische Psychotechnologien als Lösung eines uralten Menschheitsproblems erscheinen kann. Allerdings wird mit einer solchen Implementierung das reflexive Bewusstsein zunehmend als irrelevant erachtet. Damit wird zugleich die Möglichkeit zum politischen Handeln beschnitten, so wie Hannah Arendt und andere Theoretiker*innen, die das Politische von einer bloß auf Verwaltung reduzierten Politik grundlegend abheben, es konzipieren. Ein psychotechnologisch ausgemessenes Subjekt, das durch 
dieselben Technologien, die es überwachen, zu konformistischem Verhalten „,sanft“ angestupst wird, hat schlechte Voraussetzungen, um jene politische Urteilskraft zu kultivieren, die Arendt als Bedingung demokratischer Politik versteht (vgl. Zerilli 2018, S. 191). Ein solches Subjekt wird sich zudem schwer tun, jene Reflexions- sowie Partizipationskompetenz zu entwickeln, wie sie etwa im Rahmen eines subjekttheoretischen Modells politischer Bildung gefordert werden (Hellmuth und Klepp 2010, S. 116 ff.). Und ein solches Subjekt hat noch weniger eine Imaginationskompetenz, wie man im Anschluss an Bernard Stiegler formulieren könnte. Es ist nicht ohne weiteres in der Lage, Werte zu postulieren, die über die vom paternalistischen Rahmen gesteckten Zielvorgaben hinausgehen, weil ihm nicht jener offene affektive wie kognitive Horizont und damit gerade nicht jener psychische Freiraum gewährt wird, der einen gemeinsamen Diskurs und ein Ausverhandeln der Frage, wie man als politische Gemeinschaft miteinander leben will, erst möglich macht. Die algorithmische Gouvernmentalität und der psychotechnologische Paternalismus drohen im „,Tod der Politik“ zu kulminieren, wie Rouvroy (2020) jüngst attestierte.

Angesichts dieser rhetorischen Zuspitzung ist die Fruchtbarmachung eines Begriffs wie Psychopolitik umso dringlicher. Alexandra Rau hat dafür, wie oben gesehen, überaus wichtige Hinweise geliefert, weil sie im Gegensatz zu vielen dystopischen und ausweglos erscheinenden Narrativen auf die Ambivalenz und Offenheit dieser in ihren Dimensionen erst auszulotenden Kategorie setzt. Von Psychopolitik zu sprechen, bedeutet, so Rau, ,davon auszugehen, dass die Psyche als Diskurs und Praxis auf [...] hegemoniale Weise ins Feld der Macht eingetreten ist" (Rau 2010, S. 417). Die Psychopolitik umfasst Unterwerfung (unfreiwillige Subjektivierungen) ebenso wie Ermächtigung, insofern sie Menschen ,tatsächlich in den Status des Subjekts“ (ebd.) erhebt und ihnen zu kämpfen gestattet - und zwar sowohl für wie auch gegen bestimmte Formen der Subjektivierung. Raus Analyse mehrerer ,,psychopolitischer Linien“ sollte an dieser Stelle mit der Rede von „Fluchtlinien“ (Deleuze 1993b) gekreuzt werden. Wo und wie lassen sich also psychopolitische Fluchtlinien ausfindig machen, die aus der Festsetzung gefügiger produktiver Subjektpositionen hinausführen? Und wo und wie verbinden sich die individuellen Kämpfe zum kollektiven Suchen und Schaffen solcher Fluchtlinien, gerade angesichts der akuten Versuchung psychotechnologischer Automatisierungen?

Einen Umschlagpunkt hat auch Stiegler gesucht, der dem Verfallsnarrativ keineswegs das letzte Wort überlassen wollte. Wenn jede neue Technologie bzw. jedes neue „Pharmakon“12 auch neue Formen der Proletarisierung - im Sinne einer Auslagerung vormals verkörperten und habitualisierten Wissens - mit sich bringt (Stiegler 2018, S. 44), dann braucht es jeweils entsprechende, darauf reagierende Interventionen. Für die Digitalisierung gesellschaftlicher und psychologischer Verhältnisse gilt das umso mehr. Neben die Toxikologie muss eine Therapeutik treten, die das Problematisierte nicht ausschließt, sondern es zum Bestandteil jeweils neuer Sorgepraktiken

\footnotetext{
12 Der Derrida-Schüler Stiegler hat die Doppeldeutigkeit des Pharmakons, das gleichzeitig Heilmittel und Gift bedeutet, woran Derrida (1995) in seiner Lektüre von Platons Phaidros erinnert hat, zur Denkfigur der „Pharmakologie“ erhoben und auf alle „Supplemente“, Prothesen und Techniken ausgeweitet. Derrida wurde damit implizit zu einem Philosophen der Technik erklärt, der seine Kategorien allerdings fast vollständig aus seiner Auseinandersetzung mit der Schrift entwickelte (Stiegler 2009a). Sokrates wird damit aus Stieglers Sicht zum ersten Theoretiker der Proletarisierung.
} 
macht (vgl. ebd., S. 34). Dies wird ausdrücklich auch für die digitalen Technologien affirmiert: ,we cannot escape the question of a pharmacology and a therapeutics that places the digital becoming of the world at the very centre of what must be decided“ (ebd., S. 43f.), weshalb Stiegler Projekte wie Ars Industrialis und ein Digital Studies Network ins Leben gerufen hat, die philosophische Gegenwartsdiagnostik mit Öffentlichkeitsarbeit und Politikberatung verbinden, und dies gerade auch im Hinblick auf eine Industriepolitik, die der Automatisierung psychischer und kognitiver Prozesse entgegenwirkt. ${ }^{13}$ Damit versuchen Stiegler und seine Mitstreiter*innen, gegenüber den verhaltensökonomischen und psychotechnologischen Kurzschlüssen eine andere Perspektive zu eröffnen, die auf das politische Imaginäre setzt: in Stieglers Worten auf die Kraft „,noetischer Träume“ (Stiegler 2019, S. 193), die anders als die aus dem Unbewussten herrührenden Träume erfüllt, d.h. gestaltend umgesetzt werden können, und damit Politik in einem emphatischen Sinn erst möglich machen. Es sind diese scheinbar althergebrachten, tatsächlich aber mit progressiven politischen Kämpfen verbundenen kollektiven wie psychischen Fähigkeiten wie eben die, sich eine andere Zukunft überhaupt vorstellen zu können und Werte und Ideen (Stieglers „Konsistenzen“) zu schaffen, die so unverzichtbar sind für die unendlichen Aufgaben der Demokratisierung, der Deproletarisierung und der Metastabilisierung im gesellschaftlichen wie im ökologischen Sinn.

Auch wenn Hannah Arendt, wie eingangs erwähnt, das Ausmaß der Computerisierung und Digitalisierung nicht absehen konnte, erweist sich ihre Warnung vor der die menschliche Erfahrung so sehr verändernden Bedingung der Automatisierung als hellsichtig: Denn wenn das Denken auf das Schlussfolgern reduziert wird, wird es zu einer Funktion degradiert, ,welche die elektronischen Rechenmaschinen erheblich besser, schneller und reibungsloser vollziehen als das menschliche Gehirn“ (Arendt 2001, S. 410). Fasst man das Schlussfolgern als Prozessieren von Informationen auf, das von Prozessoren bewerkstelligt wird, trifft Arendts Diagnose zu. Schon sie erkannte in dieser Automatisierung die Umsetzung der Theorien des Behaviorismus, die sich ,,als nur zu richtig erweisen könnten“, indem sie beschreiben, ,was in der modernen Gesellschaft wirklich vorgeht“. In dieser Hinsicht sei es aber denkbar, dass die Arbeitsgesellschaft ,schließlich in der tödlichsten, sterilsten Passivität enden wird, die die Geschichte je gekannt hat“ (ebd., S. 411). Der Tod der Politik ist auch bei Arendt die unerfreuliche Konsequenz, wenn der Prozess der Automatisierung das psychische Leben widerstandslos in Beschlag nimmt.

Aus all dem folgt, dass die politische Theorie und insbesondere die Demokratietheorie sich verstärkt mit der Frage nach den medialen Bedingungen politischen Handelns auseinandersetzen muss - und zwar ganz besonders mit den digitalen Bedingungen und Subjektivierungen sowie der Art und Weise, wie diese politische Subjekte (re-)konstituieren. Dabei könnte sichtbar werden, dass die Digitalisierung durch die automatisierten Psychotechnologien zu einer Schwächung demokratiepolitischer Subjektformierung führt und damit etwa die Fähigkeit zur Deliberation, zur Imagination eines Gemeinwohls und der daraus gespeisten Willensbildung und demokratischen Partizipation beschneidet. Anstatt politische Subjekte zu konstituieren, die sich am Gemeinwohl orientieren und willens und in der Lage sind, über

13 Siehe http://arsindustrialis.org/lassociation (abgerufen am 14.06.2021). 
seine Ausgestaltung in einen gemeinsamen Reflexionsprozess zu treten, besteht die Gefahr, dass immer apolitischere Subjekte geformt werden, die vom digitalen Kapitalismus dermaßen vereinnahmt sind, dass ihnen schon die Idee einer radikaldemokratischen Ausverhandlung gemeinsamer Werte und Güter fremd erscheint. Wenn diese Tendenz widerstandslos hingenommen wird, dann könnte es um die Demokratie und ihre Theorie bald schon dauerhaft ruhig werden.

Danksagung Der Autor bedankt sich bei den Herausgeber*innen und den anonymen Gutachter*innen für die überaus hilfreichen Kommentare.

Funding Open Access funding enabled and organized by Projekt DEAL.

Open Access Dieser Artikel wird unter der Creative Commons Namensnennung 4.0 International Lizenz veröffentlicht, welche die Nutzung, Vervielfältigung, Bearbeitung, Verbreitung und Wiedergabe in jeglichem Medium und Format erlaubt, sofern Sie den/die ursprünglichen Autor(en) und die Quelle ordnungsgemäß nennen, einen Link zur Creative Commons Lizenz beifügen und angeben, ob Änderungen vorgenommen wurden.

Die in diesem Artikel enthaltenen Bilder und sonstiges Drittmaterial unterliegen ebenfalls der genannten Creative Commons Lizenz, sofern sich aus der Abbildungslegende nichts anderes ergibt. Sofern das betreffende Material nicht unter der genannten Creative Commons Lizenz steht und die betreffende Handlung nicht nach gesetzlichen Vorschriften erlaubt ist, ist für die oben aufgeführten Weiterverwendungen des Materials die Einwilligung des jeweiligen Rechteinhabers einzuholen.

Weitere Details zur Lizenz entnehmen Sie bitte der Lizenzinformation auf http://creativecommons.org/ licenses/by/4.0/deed.de.

\section{Literatur}

Abbinnett, Ross. 2017. The thought of Bernard Stiegler. Capitalism, technology and the politics of spirit. London, New York: Routledge.

Arendt, Hannah. 2001. Vita activa oder Vom tätigen Leben. München: Piper.

Arsène, Séverine. 2019. China's social credit system. A chimera with real claws. Asie.Visions. Ifri $110 .$.

Berg, Sebastian, Niklas Rakowski, und Thorsten Thiel. 2020. Die digitale Konstellation. Eine Positionsbestimmung. Zeitschrift für Politikwissenschaft 30:171-191.

Bogost, Ian. 2007. Persuasive games. The expressive power of Videogames. Cambridge: MIT Press.

Borucki, Isabell, und Michael Oswald. 2020. Die Vision der Digitaldemokratie und die Realität - Versuch über einen Dialog. In Demokratietheorie im Zeitalter der Frühdigitalisierung, Hrsg. Michael Oswald, Isabell Borucki, 3-15. Wiesbaden: Springer.

Bösel, Bernd. 2020. Affective media regulation, or: how to counter the blackboxing of emotional life. In Affective transformations. Politics—algorithms-media, Hrsg. Bernd Bösel, Serjoscha Wiemer, 51-70. Lüneburg: meson press.

Bösel, Bernd, und Serjoscha Wiemer. 2020. Affective transformations. Politics-algorithms-media. Lüneburg: meson press.

Busby, Mattha. 2018. Social media copies gambling methods ,to create psychological cravings'. The Guardian, 08.05.2018. https://www.theguardian.com/technology/2018/may/08/social-media-copiesgambling-methods-to-create-psychological-cravings. Zugegriffen: 19. Juli 2021.

Castel, Francoise, Robert Castel, und Anne Lovell. 1982. Psychiatrisierung des Alltags. Produktion und Vermarktung der Psychowaren in den USA. Frankfurt am Main: Suhrkamp.

Davies, William. 2016. The happiness industry. How the government and big business sold us well-being. London, New York: Verso.

Deleuze, Gilles. 1993a. Postskriptum über die Kontrollgesellschaften. In Unterhandlungen. 1972-1990, 254-262. Frankfurt am Main: Suhrkamp.

Deleuze, Gilles. 1993b. Kontrolle und Werden. In Unterhandlungen. 1972-1990, 243-253. Frankfurt am Main: Suhrkamp.

Derrida, Jacques. 1995. Platos Pharmazie. In Dissemination, 69-190. Wien: Passagen. 
Distelmeyer, Jan. 2017. Machtzeichen. Anordnungen des Computers. Berlin: Bertz + Fischer.

Eyal, Nir. 2014. Hooked. How to build habit-forming products. New York: Penguin.

Feldman Barrett, Lisa. 2017. How emotions are made: the secret life of the brain. New York: Houghton Mifflin Harcourt.

Fogg, B.J. 2003. Persuasive technology. Using computers to change what we think and do. San Francisco: Morgan Kaufmann.

Foucault, Michel. 2005. Schriften in vier Bänden. Band IV. 1980-1988. Frankfurt am Main: Suhrkamp.

Govrin, Jule. 2020. Begehren und Ökonomie. Eine sozialphilosophische Studie. Berlin, Bosten: De Gruyter.

Groot, Gerry. 2020. Schemes, Dreams, and Nightmares: China’s Paradox(es) of Trust. In China Story Yearbook: China Dreams, Hrsg. Jane Golley, Linda Jaivin, Ben Hillmann, S. 199-212. Acton: Australian National University Press.

Kahneman, Daniel. 2012. Schnelles Denken, langsames Denken. München: Siedler.

Han, Byung-Chul. 2014. Psychopolitik. Neoliberalismus und die neuen Machttechniken. Frankfurt am Main: S. Fischer.

Hayles, N. Katherine. 2017. Unthought. The power of the cognitive non-conscious. Chicago: University of Chicago Press.

Hellmuth, Thomas, und Cornelia Klepp. 2010. Politische Bildung. Geschichte-Modelle-Praxisbeispiele. Köln: Böhlau.

Hörl, Erich. 2011. Die technologische Bedingung. Berlin: Suhrkamp.

Illouz, Eva. 2007. Gefuihle in Zeiten des Kapitalismus. Frankfurt am Main: Suhrkamp.

Lewis, Paul. 2017. 'Our minds can be hijacked.' The tech insiders who fear a smartphone dystopia. The Guardian, 06.10.2017. https:/www.theguardian.com/technology/2017/oct/05/smartphoneaddiction-silicon-valley-dystopia. Zugegriffen: 19. Juli 2021.

McStay, Andrew. 2018. Emotional AI. The rise of empathic media. Los Angeles: SAGE.

Morozov, Evgeny. 2013. To save everything, click here. The folly of technological solutionism. New York: Public Affairs.

Mühlhoff, Rainer. 2018. Digitale Entmündigung und User Experience Design. Wie digitale Geräte uns nudgen, tracken und zur Unwissenheit erziehen. Leviathan 46(4):551-574.

Nosthoff, Anna-Verena, und Felix Maschewski. 2019. Die Gesellschaft der Wearables. Berlin: Nicolai Publishing.

Oswald, Michael. 2020. Digitale Disruption: Demokratietheorie im Paradigma der entgrenzten Individualkommunikation. In Demokratietheorie im Zeitalter der Frühdigitalisierung, Hrsg. Michael Oswald, Isabell Borucki, 325-345. Wiesbaden: Springer.

Pentland, Alex. 2014. Social physics. How social networks can make us smarter. New York: Penguin.

Picard, Rosalind. 1997. Affective Computing. Cambridge, MA: MIT Press.

Pritz, Sarah Miriam. 2016. Mood Tracking. Zur digitalen Selbstvermessung der Gefühle. In Lifelogging, Hrsg. Stefan Selke, 127-150. Wiesbaden: Springer.

Rau, Alexandra. 2010. Psychopolitik. Macht, Subjekt und Arbeit in der neoliberalen Gesellschaft. Frankfurt am Main: Campus.

Ross, Daniel. 2018. Introduction. In The Neganthropocene, Hrsg. Bernard Stiegler, 7-32. London: Open Humanities.

Rössler, Beate. 2017. Autonomie. Ein Versuch über das gelungene Leben. Berlin: Suhrkamp.

Rouvroy, Antoinette. 2013. The end(s) of critique. Data behaviorism vs. Due-process. In Privacy, Due process and the computational turn. The philosophy of law meets the philosophy of technology, Hrsg. Mireille Hildebrandt, Katja de Vries, 143-167. Abingdon: Routledge.

Rouvroy, Antoinette. 2020. Algorithmic governmentality and the death of politics. An interview with A. R. https://www.greeneuropeanjournal.eu/algorithmic-governmentality-and-the-death-of-politics/. Zugegriffen: 19. Juli 2021.

Sánchez-Monedero, Javier, und Lina Dencik. 2019. The politics of deceptive borders: 'Biomarkers of deceit' and the case of iBorderCtrl. arXiv. arxiv.org/abs/1911.09156. Zugegriffen: 12. August 2021

Schrape, Niklas. 2014. Gamification and Governmentality. In Rethinking Gamification, Hrsg. Mathias Fuchs, Sonia Fizek, Paolo Ruffino, und Niklas Schrape, 21-46. Lüneburg: meson press.

Slunecko, Thomas, und Laisha Chlouba. 2021. Meditation in the age of its technological mimicry. A dispositiv analysis of mindfulness applications. International Review of Theoretical Psychologies. https://doi.org/10.7146/irtp.v1i1.127079

Sonntag, Michael. 1988. Die Seele als Politikum. Psychologie und die Produktion des Individuum. Berlin: Reimer.

Stark, Luke. 2018. Algorithmic psychometrics and the scalable subject. Social Studies of Science 48(2):204-231. 
Stiegler, Bernard. 2008. Logik der Sorge. Verlust der Aufklärung durch Technik und Medien. Frankfurt am Main: Suhrkamp.

Stiegler, Bernard. 2009a. Derrida und die Technologie. In Denken bis an die Grenzen der Maschine, 111-153. Zürich, Berlin: Diaphanes.

Stiegler, Bernard. 2009b. Von der Biopolitik zur Psychomacht. Die Logik der Sorge I.2. Frankfurt am Main: Suhrkamp.

Stiegler, Bernard. 2009c. Denken bis an die Grenzen der Maschine. Zürich, Berlin: Diaphanes.

Stiegler, Bernard. 2010a. Hypermaterialität und Psychomacht. Zürich: diaphanes.

Stiegler, Bernard. 2010b. For a new critique of political economy. Cambridge: Polity Press.

Stiegler, Bernard. 2011. The decadence of industrial democracies. Disbelief and discredit. Bd. 1. Cambridge: Polity Press.

Stiegler, Bernard. 2013. What makes life worth living. On pharmacology. Cambridge: Polity Press.

Stiegler, Bernard. 2016. Digital knowledge, obsessive computing, short-termism and need for a negentropic web. In Digital humanities and digital media: interviews on politics, culture, aesthetics, and literacy, Hrsg. Roberto Simanowski, 290-304. London: Open Humanities Press.

Stiegler, Bernard. 2018. The neganthropocene. London: Open Humanities.

Stiegler, Bernard. 2019. The Age of disruption. Technology and madness in computational capitalism. Cambridge: Polity Press.

Stiegler, Bernard. 2020. Industrie der Spuren. In Milieu Fragmente. Technologische und ästhetische Perspektiven, Hrsg. Rebekka Ladewig, Angelika Seppi. Leipzig: Spector Books.

Thaler, Richard, und Cass Sunstein. 2009. Nudge. Wie man kluge Entscheidungen anstößt. Berlin: Econ.

Tufekci, Zeynep. 2014. Engineering the public: big data, surveillance and computational politics. First Monday. https://doi.org/10.5210/fm.v19i7.4901

Turkle, Sherry. 2019. Empathie-Maschinen. Der vergessene Körper. Psyche. Zeitschrift für Psychoanalyse und ihre Anwendungen 73:726-743.

Weigel, Sigrid. 2020. Der konventionelle Code als buckliger Zwerg im Dienste der Emotion Recognition. Überlegungen zu einer Urgeschichte der digitalen Kultur. Jahrbuch für Medienphilosophie 6(1):47-79.

Weinmann, Markus, Christoph Schneider, und Jan vom Brocke. 2016. Digital nudging. Business \& Information Systems Engineering 58(6):433-436.

Zerilli, Linda M.G. 2018. Toward a democratic theory of judgment. In Judgment and action: fragments toward a history, Hrsg. Soni Vivasvan, Thomas Pfau, 191-222. Evanston: Northwestern University Press.

Zuboff, Shoshana. 2018. Das Zeitalter des Überwachungskapitalismus. Frankfurt am Main, New York: Campus.

Zündel, Jana. 2017. Netflix und die Remediatisierung des Fernsehens auf Streaming-Plattformen. Montage AV 2017/1:29-47. 